\title{
SolAssist Learning: formação em tecnologias assistivas através de um MOOC e uma biblioteca virtual de soluções assistivas
}

\author{
Title: Solassist Learning: training in assistive technologies through a MOOC and a virtual library \\ of assistive solutions
}

\author{
Rosana Wagner \\ Universidade Federal do Rio Grande do Sul (UFRGS) \\ Centro Interdisciplinar de Novas Tecnologias na Educação \\ (CINTED) - Porto Alegre, RS - BRASIL \\ rosanawagner@gmail.com
}

\section{Liliana Passerino}

Universidade Federal do Rio Grande do Sul (UFRGS)

Centro Interdisciplinar de Novas Tecnologias na Educação (CINTED) - Porto Alegre, RS - BRASIL

lpasserino@gmail.com

\author{
Roberto Franciscatto \\ Universidade Federal do Rio Grande do Sul (UFRGS) \\ Centro Interdisciplinar de Novas Tecnologias na Educação \\ (CINTED) - Porto Alegre, RS - BRASIL \\ roberto.franciscatto@gmail.com \\ José Valdeni de Lima \\ Universidade Federal do Rio Grande do Sul (UFRGS) \\ Centro Interdisciplinar de Novas Tecnologias na Educação \\ (CINTED) - Porto Alegre, RS - BRASIL \\ valdeni@inf.ufrgs.br
}

\section{Sidnei Silveira}

Dr. Sidnei Renato Silveira

Universidade Federal de Santa Maria

Frederico Westphalen - RS, Brasil

sidneirenato.silveira@gmail.com

\begin{abstract}
Resumo O trabalho em questão visa descrever sobre a formação em tecnologias assistivas, através da utilização de um MOOC projetado e estruturado para este fim, bem como, uma biblioteca virtual de soluções assistivas projetada e desenvolvida, como ferramenta de apoio a formação proposta. $O$ artigo apresenta os principais conceitos acerca das tecnologias educacionais e assistivas, seguido dos trabalhos relacionados a MOOC e as bibliotecas virtuais assistivas. A seguir, a metodologia empregada neste trabalho é exposta, bem como, o processo de desenvolvimento do MOOC, sua estrutura, proposta e objetivos, seguido da arquitetura da biblioteca virtual de soluções assistivas, tecnologias utilizadas e princípios básicos de funcionamento. Por fim, são apresentados os resultados obtidos com as formações realizadas até o presente momento, além dos trabalhos futuros, como forma de incrementar e aperfeiçoar o projeto, baseado nas respostas dos participantes do mesmo. O grande diferencial do trabalho está no fato de o mesmo utilizar-se de tecnologias educacionais abertas, como os MOOCs e o potencial que os mesmos provém, unindo isto a uma biblioteca virtual de soluções assistivas própria, projetada e desenvolvida sob o contexto da educação inclusiva, formando um projeto único, útil e dinâmico a formação em tecnologias assistivas.
\end{abstract}

Palavras-Chave: MOOCs, Tecnologia Assistiva, Solassist, Biblioteca Virtual, Acessibilidade.
Abstract
The work in question aims to describe on training in assistive technologies through the use of a MOOC designed and structured for this purpose, as well as a virtual library of assistive solutions designed and developed as a support tool the proposed training. The article presents the main con- cepts about educational and assistive technologies, followed by work-related MOOC and assistive virtual libraries. Next, the methodology used in this work is exposed, as well as the development process of the MOOC, its structure, and proposed objectives, followed by the architecture of the 
virtual library of assistive solutions, technologies used and basic principles of operation. Finally, the results obtained with the training undertaken to date are presented in addition to the future work as a way to enhance and improve the project, based on the responses of participants. The great work of the differential is in fact the same use is open educational technologies such as MOOCs and the potential that the same comes, linking it to a virtual library of own assistive solutions, designed and developed in the context of inclusive education, forming a single, useful and dynamic training in assistive technology project.

Keywords: MOOCs, Assistive Technology, Solassist, Virtual Library, Accessibility 


\section{Introdução}

A Educação a Distância (EAD) evoluiu desde a década de 90, passando para Ambientes Virtuais de Aprendizagem (AVAs) muito populares como o Moodle, Teleduc, Rooda, entre outros. No final de 2011 surgem os MOOCs, os quais compreendem um tipo de Curso Aberto desenvolvido por meio da utilização de AVAs e de ferramentas como a Web 2.0. Estes, apresentam um novo cenário para a $\mathrm{EAD}$, no que se refere à transição da lógica da transmissão para a lógica da comunicação (interatividade) entre os mais diversos perfis de usuários. Têm seus pilares fundamentados na democratização do acesso a conteúdos educacionais.

Os MOOCs compreendem uma metodologia de aprendizagem, sendo assim independentes de plataforma. Atualmente têm sua oferta concentrada no cenário educacional americano, tendo como principais expoentes: Coursera, Udacity, EDX [7].

No que se refere ao panorama educacional brasileiro em EAD, os MOOCs representam uma mudança nos processos de aprendizagem online, intensificando a aprendizagem, colaboração e interação, troca de ideias, dúvidas, compartilhamento de experiências e construção coletiva entre os estudantes. A interatividade e a utilização de mídias educativas são fundamentais nos MOOCs.

A proposta apresentada neste trabalho visa integrar o poder de interação e colaboração dos MOOCs com a capacitação destes usuários junto as Tecnologias Assistivas (TA) utilizando como ferramenta de apoio a este objetivo uma biblioteca virtual de soluções assistivas própria, desenvolvida pelos autores deste trabalho de pesquisa, denominada SolAssist. A integração destes recursos tecnológicos, bem como, a originalidade da ideia, provê um sistema hierárquico dinâmico e prático ao aprendizado sobre as TAs e suas múltiplas utilizações no dia-a-dia.

Desta forma este trabalho encontra-se organizado da seguinte forma: no segundo capítulo apresenta-se as tecnologias educacionais e assistivas que dão suporte a este artigo, através das quais foi possível organizar um MOOC de Tecnologia Assistiva. O conceito de MOOCs é apresentado, bem como quais são as principais características de criação de um MOOC.

O terceiro compõe trabalhos relacionados que estão sendo realizados através da utilização de diferentes plataformas e tecnologias.

A metodologia de desenvolvimento desta pesquisa é apresentada no quarto capítulo, e a seguir, no quinto apresenta-se o projeto piloto realizado através da integra- ção do MOOC Solassist Learning com a biblioteca de soluções assistivas Solassist. Os resultados do projeto piloto estão apresentados no capítulo 6 e os trabalhos futuros no capítulo 7 .

\section{Tecnologias educacionais e assisti- vas}

Apesar de ser um assunto largamente discutido e aprimorado na atualidade, a acessibilidade vem sendo desenvolvida desde os primórdios da humanidade, quando as pessoas resolviam dificuldades relativas à deficiência utilizando os recursos que possuíam. Muito antes do surgimento do termo TA, as práticas de acessibilidade já existiam, efetivamente, sob a forma de simples instrumentos que melhoravam a autonomia da pessoa com deficiência (PCD).

Segundo [6], pode-se denominar de tecnologia assistiva desde uma bengala, utilizada por nossos avós, como forma de proporcionar conforto e segurança no momento de seu caminhar, bem como, um aparelho de amplificação usado por uma pessoa com surdez ou mesmo um veículo adaptado para uma pessoa com deficiência.

O conceito de Tecnologia Assistiva vem sendo aprimorado, contudo, devido à infinidade de recursos que hoje são abrangidos por essa área. De acordo com [9], a TA é um termo utilizado para identificar "todo o arsenal de recursos e serviços que contribuem para proporcionar ou ampliar habilidades funcionais de pessoas com deficiência", promovendo vida independente, autonomia e inclusão. Tais recursos e serviços propostos pela Tecnologia Assistiva são respostas para qualquer incapacidade do indivíduo e podem ser compreendidos como soluções na área da acessibilidade. A partir desta análise, tem-se, então, a expressão "Soluções Assistivas", que compreendem casos de sucesso na inserção da acessibilidade nos diversos meios sociais.

As soluções assistivas não necessariamente precisam de tecnologia para serem efetivas, tendo como base estratégias a serem implantadas na sociedade em geral para promover a inclusão. Verifica-se, contudo, uma carência de soluções assistivas por parte de empresas e demais ambientes sociais, estimulada pela falta de informação e suporte na implantação e no gerenciamento destas tecnologias [6].

Dentro deste contexto, estuda-se a vantagem que as bibliotecas virtuais apresentam na divulgação de informação e troca de experiências acerca das práticas acessíveis, visto que o conteúdo nelas inserido é disponibilizado para 
qualquer usuário, em qualquer lugar e a qualquer momento. Juntamente com uma biblioteca virtual de soluções assistivas pensou-se na articulação de um curso (disponibilizado via MOOC) voltado ao treinamento sobre tecnologias assistivas, tema deste trabalho e relatado de forma mais detalhada nas seções subsequentes.

\subsection{MOOCs: Massive Open Online Courses}

Um MOOC (Massive Open Online Courses) nada mais é do que um tipo de curso aberto (permite o livre acesso, sem restrições, a oportunidades de aprendizagem) ofertado por meio de ambientes virtuais de aprendizagem (AVAs) ou outras tecnologias correlatas, objetivando disponibilizar a uma grande quantidade de alunos a oportunidade de ampliar seus conhecimentos em um processo de coprodução.

As características que configuram os MOOCs são a de serem: cursos abertos e que permitem escalabilidade. Sobre a primeira, significa que mesmo estudantes que não estão regularmente registrados na instituição promotora podem participar. No entanto, é preciso lembrar que uma limitação ao aberto está associada à exigência de habilidades mínimas por parte dos participantes, o letramento digital, além da infraestrutura tecnológica com acesso à internet e preferencialmente com uma banda larga de qualidade que permita a navegação sem muitas frustrações. Sobre escalabilidade, o desenho do curso é apropriado para atender crescimento exponencial de matrículas, podendo chegar a centenas de milhares de estudantes participando em cada oferta de curso [8]

A ideia dos MOOCs é, de fato, precedida pelo movimento global de Recursos Educacionais Abertos (REA) e que vem ganhando espaço no cenário nacional e internacional. REA, termo cunhado em evento da Unesco em 2002, são materiais educacionais ou de pesquisa, incluindo cursos completos, objetos de aprendizagem, textos, vídeos, livros e software, disponíveis em qualquer formato ou mídia, que estejam em domínio público ou que tenham uma licença de uso aberta, permitindo o reuso e adaptação por terceiros [8].

Neste contexto, os Recursos Educacionais Abertos (REA) são imprescindíveis, no sentido de permitir que as informações sejam livremente acessadas, reusadas, remixadas e redistribuídas. Quanto mais um curso emprega licenças restritivas em seus conteúdos, mais barreiras surgem, e assim restringem o fluxo e acesso das informações e, portanto, se distanciam-se da essência de um MOOC [12].

A integração dos MOOCs com recursos tecnológicos, como as mídias digitais, visa facilitar e disseminar a utilização destes, porém ao mesmo tempo representa uma forma de pré-requisitos para utilização desta metodologia. Ainda que a maior parte dos MOOCs possam ser considerados como em caráter de experiências piloto, em sua dinâmica atual aparece duas correntes distintas, as quais divergem-se, com baixas chances de reencontro posterior, o que difere em objetivos e métodos [3]. No que diz respeito aos ramos derivados dos MOOCS, podem ser associados dois tipos que são o cMOOCs e os xMOOCs, tais como Coursera e edX.

Os cMOOCs são baseados no Conectivismo, uma teoria da era digital que parte da premissa que o conhecimento está no mundo e não apenas no indivíduo, como afirmam outras correntes de aprendizagem, tais como o Cognitivismo e o Construtivismo [3]. Alguns elementos que ajudam a caracterizar o Conectivismo são, de acordo com [13]: i. aprendizagem e conhecimento estão diretamente associados à máxima diversidade de opiniões, ii. Aprendizagem passa por um adequado processo de conexão a fontes especializadas de informação, iii. Desenvolvimento da capacidade de saber mais é mais relevante do que o que momentaneamente se sabe, iv. Capacidade de enxergar conexões entre os campos de conhecimentos, ideias e conceitos constitui uma habilidade central, e v. aprendizagem, nesta modalidade, depende de máquinas conectadas, demais infraestrutura tecnológica associada e facilidades de conexão como elementos essenciais para facilitar uma aprendizagem que possa fluir sem dificuldades.

Assim, os cMOOCs se caracterizam pela utilização do conteúdo como ponto de partida e os estudantes são encorajados a expandir e criar conhecimentos a partir de seus próprios interesses e buscas na rede. Via os processos de agregação, mixagem, customização e compartilhamento, os cursos evoluem. Agregação significa que, diferentemente dos cursos tradicionais nos quais o conteúdo é estático e definido preliminarmente, durante o curso, após o seu início, novos conteúdos são permanentemente agregados ao ecossistema do curso, baseado na interatividade entre os professores responsáveis e os estudantes participantes, todos entendidos como contribuintes ao cMOOC [8].

Mixagem porque é fortemente estimulado associar o material específico preparado para o curso com outros conteúdos que forem tornados disponíveis ao longo do curso. Customização, desde que o material resultante da mixagem seja permanentemente redesenhado em uma forma customizada tal que seja apropriadamente utilizado pelos participantes, viabilizando que eles atinjam seus objetivos específicos. Compartilhamento é associado ao fato que o material redesenhado deve ser distribuído a todos os demais participantes do curso. Ou seja, um cMOOC utiliza múltiplas plataformas (blogs, wikis, websites e redes sociais as mais variadas), além de priorizar a 
interatividade entre os participantes. O cMOOC, portanto, não é todo planejado desde o início, a experiência evolui conforme o curso se desenvolve [8].

Os xMOOCS, diferentemente dos cMOOCS, são mais recentes ainda e se baseiam num formato estruturado e tradicional, que segue um fluxo, a partir de conhecimento pré-definido pelo professor, compartilhado de um para muitos. Neste modelo a figura do professor é central e em geral professores doutores de universidades renomadas os responsáveis, os conteúdos são definidos a priori pela instituição e não se prioriza a interatividade entre os participantes [8].

Com base no contexto deste capítulo, após a apresentação de um dos mais tradicionais ambientes de aprendizagens, o MOODLE, insere-se o contexto dos MOOCs que possibilitam uma nova metodologia de formação, a qual busca integrar conteúdos mais interativos e dinâmicos, como conexão com servidores hospedeiros de ambientes de realidade virtual, a utilização de plataformas diferenciadas e de OAs interativos. A metodologia dos MOOCs visa a utilização de interação através de mídias interativas.

\subsection{Processo de desenvolvimento de um MOOC}

O processo de desenvolvimento de um MOOC, inicialmente pode ser considerado semelhante ao de um curso de EAD. Os cursos de EAD geralmente utilizam AVAs. Os MOOC também podem utilizar os AVAs, porém geralmente os MOOCs são desenvolvidos sob plataformas desenvolvidas especificamente para a criação do mesmo.

Outra característica dos MOOCs é terem recursos educacionais abertos ou objetos de aprendizagem, estes diminuem a quantidade de leitura que o aluno deve realizar, bem como tornam o aprendizado mais dinâmico e atrativo.

Os MOOCs, como seu próprio nome diz, são desenvolvidos para um grupo massivo de estudantes, ou seja, não há tanta interação com o professor, os alunos trocam experiências entre si, bem como, não ocorrem correções de atividades descritivas e longas.

Por esse motivo, os MOOCS não são utilizados para cursos de longa duração, como cursos de graduação ou pós-graduação, mas sim para cursos de 30 a 200 horas aproximadamente e são destinados a pessoas que desejam saber um pouco mais sobre um assunto, porém não constituem uma formação completa.

Para que seja possível desenvolver um MOOC, primeiramente é necessário ter um assunto bem desenvolvido e com bastante material didático (de preferência dinâ- mico), planejar uma carga horária que seja possível o desenvolvimento do conteúdo previsto e um ambiente tecnológico capaz de hospedar os conteúdos e que permita que os usuários realizem cadastros e possam interagir no ambiente com outros colegas.

Para o desenvolvimento destes MOOCs uma vasta gama de tecnologias pode ser utilizada. A seguir são apresentados alguns dados a respeito da EAD e dos MOOCs e as tecnologias que podem ser adotadas para o desenvolvimento desta modalidade

\section{Trabalhos relacionados}

Nesta seção são apresentados alguns dos principais trabalhos relacionados, com relação ao objetivo proposto para este artigo. A ferramenta exposta na seção 3.1 corresponde a um ambiente de MOOC voltado ao ensino de tecnologia assistiva em geral, enquanto que na seção 3.2, são apresentados os principais portais de tecnologias assistivas, na ideia de ferramentas que podem ser utilizadas para descrever, expor e caracterizar tecnologias assistivas na prática, fazendo um contraponto com a biblioteca virtual de soluções assistivas (SolAssist) desenvolvida para este fim.

\subsection{Plataforma SiestaTV Learning}

A SiestaTV Learning é um AVA, porém com o conceito voltado para cursos dinâmicos e que utilizem mais recursos didáticos, como vídeos interativos. Esta plataforma é desenvolvida pela empresa GetitCorp. [5]

A SiestaTV Learning visa desenvolver cursos de acordo com o formato de MOOCs. É uma plataforma com tecnologia acessível, simples, interativa, móvel utilizando recursos $3 \mathrm{D}$, a qual é possível acessá-la através da televisão, computador, notebook, tablet ou smartphone. [5]

Esta plataforma tem grande interatividade uma vez que pode ser utilizada através de interação com Unity (realidade virtual) onde são desenvolvidos jogos e perguntas de interação. Atualmente esta plataforma é utilizada basicamente para a formação de professores em mídias interativas.

O ambiente é desenvolvido com base em vídeos, cuja estrutura sequencial pode remeter a diferentes tipos de mídias, ressaltando que esse avanço na estrutura de conteúdo somente poderá ocorrer após o término da visualização do vídeo. Entre cada vídeo há uma atividade, baseada no conteúdo que foi demonstrado no vídeo. A 
Figura 1 apresenta a tela inicial do ambiente desenvolvido, onde é possível acessar a canais de TV, fóruns, chats, mensagens e aos cursos nos quais está cadastrado.

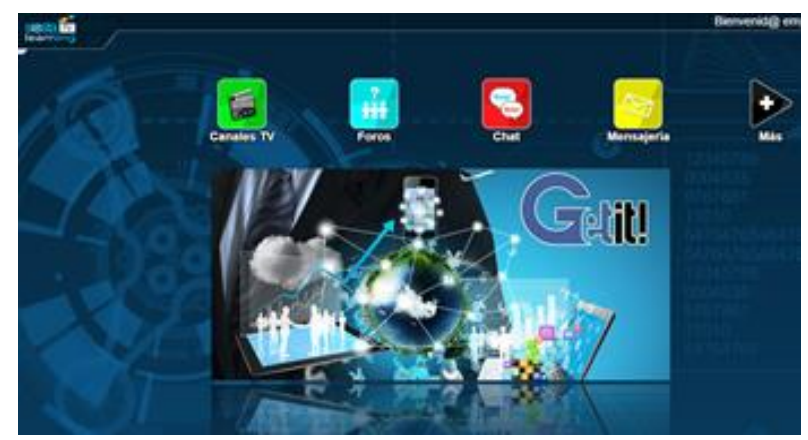

Figura 1 - Tela inicial da plataforma SiestaTv Learning. Fonte:[5]

Este ambiente é considerado muito interessante ao se tratar de cursos como MOOCs, que contenham conteúdos mais dinâmicos e que necessitam de uma maior interação e interatividade com os usuários. Considera-se que seja um ambiente que visa a interação entre o usuário e a plataforma, estando assim de acordo com o conceito de MOOCs.

\subsection{Portais de Tecnologias Assistivas como alternativa de apoio a MOOCs}

Existem atualmente diversos portais de tecnologia assistiva que podem se mostrar úteis quando pretende-se utilizar estes, como ferramenta de suporte e apoio ao ensino e exemplificação de tecnologias assistivas. As ferramentas apresentadas abaixo, são exemplos de portais sobre tecnologias assistivas espalhados pelo mundo.

A. AAATE (Association for the Advancement of Assistive Technology in Europe)

A AAATE (em português, Associação para o Avanço da Tecnologia Assistiva na Europa), é uma associação pan-europeia interdisciplinar dedicada aos aspectos da tecnologia assistiva, como o uso da mesma, pesquisa, desenvolvimento, manufatura, suprimentos e políticas.

Possui atualmente mais de 250 membros e tem como missão estimular o avanço da tecnologia assistiva para o benefício das pessoas com deficiência, incluindo pessoas idosas. Entre as principais atividades desenvolvidas pela AAATE estão: organização de conferências internacionais sobre tecnologia assistiva; revista científica da AAATE; seminários, workshops e eventos de informação [1].

B. EASTIN (European Assistive Technology Information Network)
O EASTIN (http://www.eastin.eu) é um motor de busca europeu que fornece acesso a recursos de Tecnologia Assistiva. São mais de 50.000 produtos disponibilizados no portal, 5.000 empresas produtoras e distribuidoras, permitindo ao usuário realizar buscas completas e navegar por entre notícias, estudos de caso e demais informações relativas à acessibilidade.

Através do EASTIN, pessoas que utilizam a tecnologia assistiva, profissionais de saúde, gestores e demais interessados podem encontrar sugestões que auxiliem na resolução de problemas de autonomia no cotidiano de pessoas com deficiência [3].

\section{Portal Nacional de Tecnologia Assistiva}

Portal brasileiro mantido pelo Instituto de Tecnologia Social (ITS - http://www.assistiva.org.br/), criado com o objetivo de oferecer informações para o incentivo de políticas públicas que promovam a inclusão social e melhoria de vida dos cidadãos.

Este portal concentra material sobre inúmeros tipos de deficiência, além de notícias, legislação e catálogo de produtos relacionados à acessibilidade, permitindo tornar as soluções mais acessíveis para o benefício de todas as pessoas que delas necessitam. [10].

\section{SIVA}

O SIVA (http://portale.siva.it/) é um portal italiano que fornece orientações ao público acerca dos benefícios que a tecnologia traz na qualidade de vida e participação de pessoas com deficiência. Através de cadastros de produtos assistivos realizados neste portal, os usuários podem ter acesso a informações consistentes que auxiliam nas práticas de inclusão social e digital.

O SIVA faz parte da rede EASTIN, citada anteriormente e é dedicado a qualquer pessoa interessada na área da Tecnologia Assistiva, seja profissional, portador de deficiência ou pesquisador. [11].

Vale salientar que a principal diferença entre as soluções apresentadas nesta seção e o protótipo desenvolvido neste trabalho (SolAssist) como suporte ao curso proposto via MOOCs, se dá pela proposta da biblioteca virtual de soluções assistivas que é de coletar soluções assistivas que são utilizadas na prática laboral no dia-adia de pessoas com deficiência. Estas soluções podem ser inseridas na biblioteca tanto por pessoas físicas, quanto empresas que fazem uso ou desenvolveram soluções assistivas para determinado contexto, colaborando para a inclusão de pessoas com deficiência no mercado de trabalho de forma efetiva. Outro diferencial são os recursos de acessibilidade, usabilidade e design responsivo que ga- 
rantem a biblioteca ser acessada por pessoas com diferentes necessidades especiais, bem como, sua disponibilização a diferentes dispositivos eletrônicos em que a mesma pode ser acessada e utilizada.

\section{Metodologia}

O artigo aqui descrito é originário de um projeto de pesquisa intitulado Solassist Learning. Este por sua vez, caracteriza-se como um subprojeto do Projeto Solassist (principal). Com início em julho de 2012, o Solassist é o mais recente projeto de Pesquisa do Grupo TEIAS1.

O objetivo principal do projeto Solassist é desenvolver uma Biblioteca Virtual de Soluções Assistivas, com acesso livre e gratuito, que possibilite, além da consulta por parte de usuários e comunidade, atender questões de formação continuada e servir de base para tomada de decisões na implantação e implementação de políticas públicas. Este projeto está alicerçado no entendimento de que a sistematização e divulgação das tecnologias assistivas poderá facilitar e socializar as soluções de pessoas com deficiência ou dificuldades diversas, nas tarefas do cotidiano, principalmente no trabalho. Desta forma, o projeto Solassist tem por objetivo identificar, organizar, categorizar e divulgar soluções de tecnologias assistivas em uso, além de formar gestores para o atendimento destas necessidades nas organizações produtivas do país.

A pesquisa realizada no projeto Solassist Learning, conforme já apresentado anteriormente, tem como problema de investigação: como estruturar/organizar um MOOC de TA que apoie o processo de formação de professores do Ensino Profissionalizante que atuam na preparação para a inclusão de PCD no mercado de trabalho.

Trata-se de uma pesquisa quanti-qualitativa a partir do método estudo de caso.

A pesquisa qualitativa, visa à "análise de casos concretos em suas peculiaridades locais e temporais, partindo das expressões e atividades das pessoas em seus contextos locais". [1] Justifica-se a pesquisa qualitativa neste trabalho por obtermos dados descritos por meio de sentimentos e conhecimentos expressados pelos partici-

\footnotetext{
${ }^{1}$ O Grupo de Pesquisa TEIAS - Tecnologias em Educação para Inclusão e Aprendizagem em Sociedade, coordenado pela Professora Liliana Passerino vem se dedicando sistematicamente ao ensino, extensão e à pesquisa sobre a tecnologia, linguagem e comunicação de pessoas com deficiência, em especial, com autismo, deficiência intelectual, paralisia cerebral e deficiência motora.
}

pantes no envolvimento com as atividades propostas no decorrer do curso. Dentre as técnicas possíveis a serem realizadas na pesquisa qualitativa, será utilizado o método estudo de caso exploratório e descritivo.

Esta pesquisa utilizou como base os principais autores e pesquisadores de cada uma das áreas apresentadas. A ampla pesquisa bibliográfica desenvolvida torna capaz a realização de um estudo exploratório de descritivo, tornando possível confrontar os dados obtidos com base nas teorias previamente estudadas.

Os instrumentos de coleta de dados utilizados são fóruns, entrevistas e questionários.

A pesquisa quantitativa também é utilizada nesta pesquisa e tem por finalidade computar alguns dados de questionário elaborados. A pesquisa quantitativa foi utilizada como suporte a pesquisa qualitativa e como forma de obtenção de alguns dados da pesquisa que puderam ser observados por meio deste método de pesquisa.

A triangulação entre os dados obtidos por meio das pesquisas qualitativas e quantitativas permite representar as diferentes perspectivas metodológicas para a análise de um tema, sendo este processo compreendido como a compensação complementar das deficiências e dos pontos obscuros de cada método isolado. A base desta concepção é o insight lentamente estabelecido de que "métodos qualitativos e quantitativos devem ser vistos como campos complementares e não rivais" [4].

A seguir apresentamos o projeto piloto que foi desenvolvido. Através do projeto piloto foi possível analisar dados positivos e negativos que puderam ser mantidos ou alterados para a versão final do projeto.

\section{Desenvolvimento}

Os MOOCs representam cursos de curta duração, os quais não visam uma formação completa ao cursista, mas sim possibilitam uma formação inicial sobre algum assunto específico. A principal característica é ter conteúdos dinâmicos e possibilitar uma formação através de recursos digitais. Com base nisso desenvolveu-se o MOOC Solassist Learning, exposto em maiores detalhes na seção 5.1 e também projetou-se e desenvolveu-se a biblioteca virtual de soluções assistivas (denominada SolAssist) descrita de forma mais clara na seção 5.2, bem como, sua arquitetura de funcionamento, sendo ambas tecnologias perfeitamente "casadas" para o entendimento e compreensão acerca das TAs. 


\subsection{Solassist Learning}

O projeto piloto teve início em setembro de 2014 e término em janeiro de 2015, atingindo uma carga horária de 100 horas. O público alvo foi profissionais que atuam em cursos profissionalizantes e com pessoas com deficiência matriculadas.

Os participantes foram indicados pela Auditora Fiscal do Trabalho - Ministério do Trabalho e Emprego e Coordenadora do Projeto Inclusão de Pessoas com Deficiência no Trabalho - SRTE/RS, Ana Maria Machado da Costa. Após a indicação de quais seriam as empresas e projetos mais indicados para realização do estudo, foram selecionados interessados que se manifestaram por meio de uma inscrição prévia. Na inscrição prévia, além de dados básicos dos participantes, foi solicitado também um atestado da instituição de trabalho comprovando que o interessado atua no ensino profissionalizante e que há pessoas com deficiência na sua turma ou meio de atuação. Esta seleção visou garantir que as vagas fossem destinadas especificamente para o público alvo de nosso interesse. Foram selecionados 34 participantes.

Os pré-requisitos estabelecidos para os participantes foram ser usuário das TICs (uso de editores de texto e ferramentas de imagem básicas); dispor de computador com acesso à internet (banda larga); possuir uma conta de e-mail. O curso foi organizado em sete módulos, por meio dos quais foi possível organizar os conteúdos previstos, conforme tabela 01 :

Unidade 1: INCLUSÃO: perspectivas históricas e atuais

\begin{tabular}{l|l}
\hline Duração: & 1 semana/7dias \\
\hline Carga Horária: & $07 \mathrm{hs}$ \\
\hline
\end{tabular}

Unidade 2: MARCO POLÍTICO-LEGISLATIVO

\begin{tabular}{l|l}
\hline Duração: & 1 semana/7dias \\
\hline Carga Horária: & $07 \mathrm{hs}$ \\
\hline
\end{tabular}

Unidade 3: TECNOLOGIA ASSISTIVA E ACESSIBILIDADE

\begin{tabular}{l|l}
\hline Duração: & 4 semanas/28dias \\
\hline Carga Horária: & $28 \mathrm{hs}$ \\
\hline Unidade 4: PAPEL SOCIAL DA TECNOLOGIA \\
ASSISTIVA \\
\hline Duração: & 1 semana/7dias \\
\hline Carga Horária: & 07 hs \\
\hline Unidade 5: EDUCAÇÃO PROFISSIONAL E \\
\begin{tabular}{l|l} 
INCLUSÃO \\
\hline Duração: & 2 semanas/14dias \\
\hline Carga Horária: & $14 \mathrm{hs}$ \\
\hline
\end{tabular}
\end{tabular}

Unidade 6: PRÁTICAS PEDAGÓGICAS E INSERÇÃO LABORAL COM APOIO DE TECNOLOGIAS

\begin{tabular}{l|l}
\hline Duração: & 4 semanas/28dias \\
\hline Carga Horária: & $28 \mathrm{hs}$ \\
\hline $\begin{array}{l}\text { Unidade 7- AVALIAÇÃO DO CURSO/questionário e } \\
\text { produção escrita }\end{array}$
\end{tabular}

\begin{tabular}{l|l}
\hline Duração: & 1 semana/7dias \\
\hline Carga Horária: & $09 \mathrm{hs}$ \\
\hline
\end{tabular}

Tabela 01. Organização do curso Solassist Learning

O curso foi desenvolvido na modalidade EAD, por meio do ambiente virtual de aprendizagem Wikispaces. Outras ferramentas, como o PbWorks e o Google Drive foram utilizadas.

A Figura 2 apresenta a aba "home" do curso, onde consta o logo do curso, bem como informações iniciais. Quando apresentada aos participantes as abas e os conteúdos laterais foram sendo disponibilizadas conforme o andamento dos conteúdos.

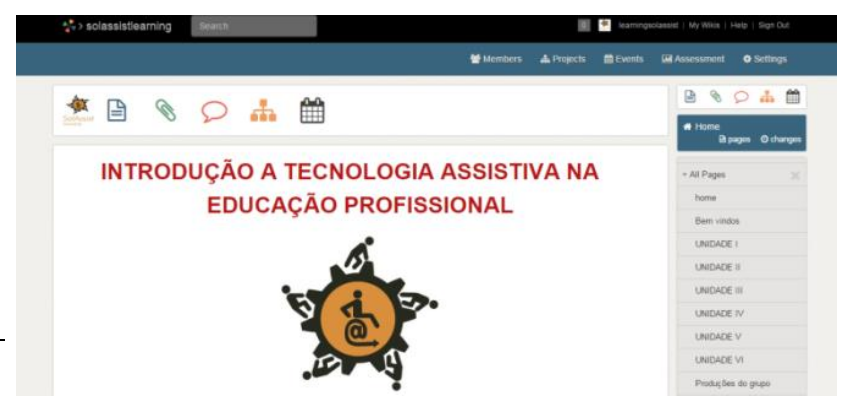

Figura 2 - Aba Home do Solassist Learning

A Figura 3 apresenta a aba Bem Vindos, na qual constam informações acerca do curso, bem como, instruções iniciais de questionários a serem respondidos antes de iniciar o curso.

Nesta aba também constam informações sobre as datas e módulos a serem estudados. Foi disponibilizado um tutorial com os passos principais para acesso ao PbWorks.

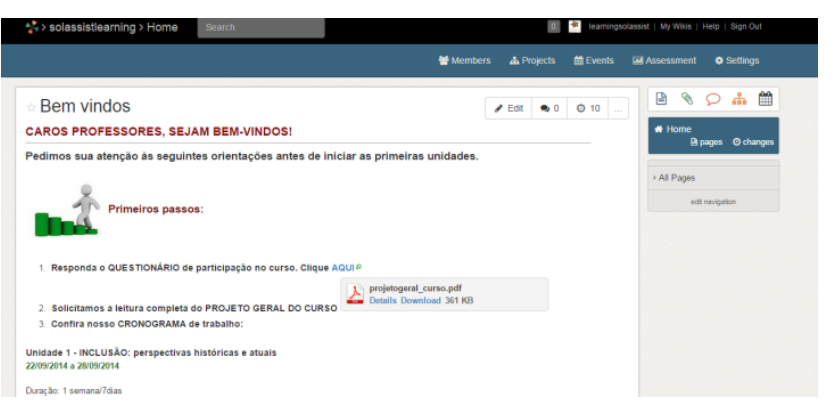


Figura 3 - Aba Bem Vindos do Solassist Learning

As Figuras 4 e 5 apresentam respectivamente as Unidades I e II, com a apresentação dos conteúdos e das atividades. $\mathrm{O}$ curso foi dividido em 7 unidades, cada uma com seus respectivos conteúdos e atividades. A maior parte das atividades foram desenvolvidas no próprio ambiente de trabalho dos participantes, possibilitando assim que tivéssemos acesso aos dados da instituição e dos procedimentos que estavam sendo aplicados.

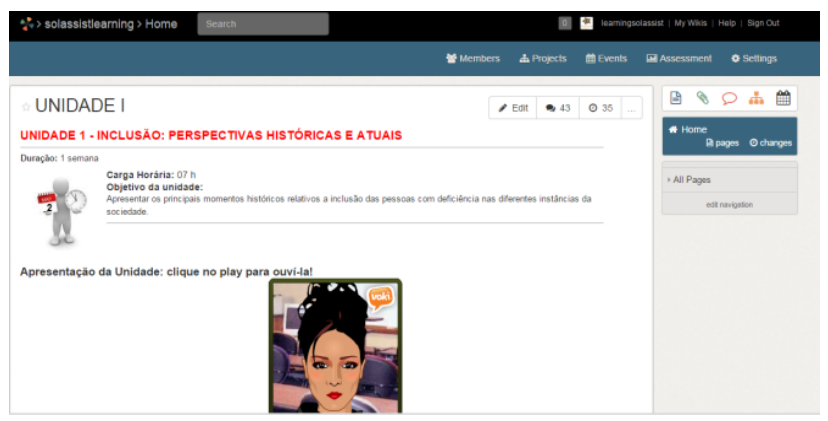

Figura 4 - Unidade I

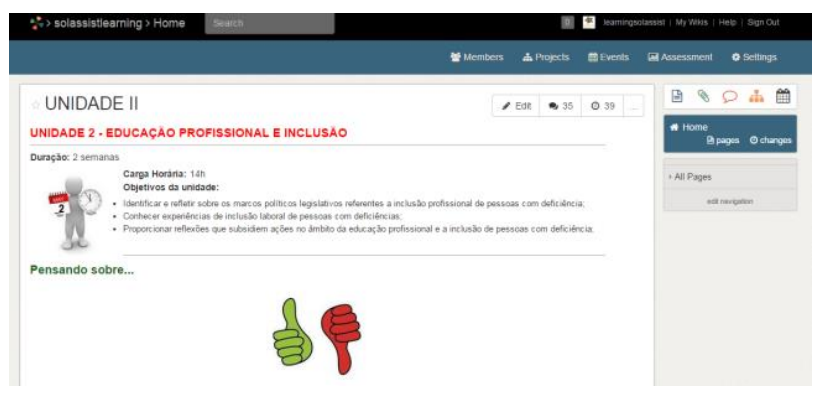

Figura 5 - Unidade II

Para que os participantes pudessem interagir, bem como aprender um com os outros, foi criada uma aba "produções do grupo", conforme Figura 6, local onde divulgou-se todos os links dos Pbworks pessoais, possibilitando assim que todos pudessem visualizar os conteúdos dos colegas, tornando assim a aprendizagem mais dinâmica e completa, por meio da interação entre os participantes.

No Wikispaces é possível restringir o acesso, desta forma foi possível controlar que todas as informações apenas pudessem ser acessadas pelos próprios participantes e pelos administradores, mantendo assim a identidade dos participantes preservada.

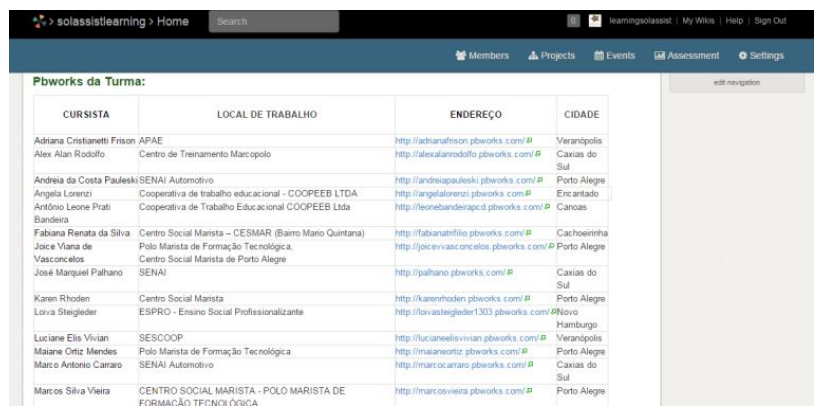

Figura 6 - Aba produções do grupo

As atividades relacionadas ao desenvolvimento de alguma atividade no ambiente escolar e após a apresentação de dados das atividades foram disponibilizadas/entregues como resposta as atividades por meio do PbWorks. Atividades relacionadas a questionários e enquetes foram realizadas por meio do Google Drive, os fóruns de participação foram realizados por meio de recursos do próprio Wikispaces.

\subsection{Arquitetura da Biblioteca Virtual de So- luções Assistivas}

A biblioteca virtual de soluções assistivas (ferramenta de apoio ao curso oferecido via MOOC), foi projetada conforme os passos definidos abaixo.

Como estágio inicial, foram analisados e identificados os requisitos básicos necessários ao desenvolvimento da biblioteca virtual. A ideia de funcionamento geral baseou-se em uma biblioteca de soluções composta por contribuições dos usuários previamente cadastrados no sistema, sejam eles pessoas físicas ou jurídicas. Os usuários, após efetuarem seu registro pessoal, podem incrementar a biblioteca cadastrando soluções assistivas, que passam a ser disponíveis para qualquer pessoa que busque informações no sistema.

A partir dos requisitos levantados para o desenvolvimento da biblioteca virtual, foi realizada a modelagem de dados, como forma de avaliar como o banco de dados deveria ser moldado para receber todas as informações necessárias ao funcionamento do website. Verificouse, então, a necessidade do sistema apresentar interfaces para cadastro e login de usuário, cadastro de soluções assistivas e principalmente, um mecanismo de busca para retornar informações de acordo com a pesquisa realizada.

As páginas da biblioteca começaram a ser produzidas com auxílio do framework Bootstrap, para que todo o conteúdo pudesse ser adaptável a diferentes dispositivos de acesso. Foram projetados os formulários para cadastro de pessoa física e jurídica, seguidos pelo formulário de login. A página inicial da biblioteca virtual de soluções assistivas, pode ser visualizada, conforme Figura 7. 


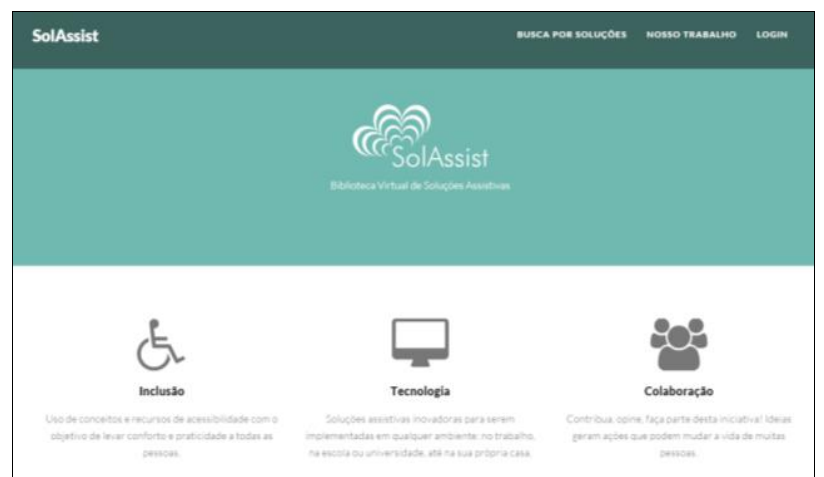

Figura. 7. Página inicial da biblioteca virtual de soluções assistivas

Uma vez realizado o cadastro, as informações do usuário armazenadas no banco de dados foram utilizadas para uma personalização de seu perfil, apresentado após autenticação na biblioteca. Neste perfil, o usuário pode visualizar suas informações pessoais, pode acessar o formulário de cadastro de soluções assistivas, bem como as soluções por ele cadastradas.

Ao terminar o desenvolvimento do cadastro de soluções, deu-se início a uma segunda etapa de desenvolvimento, na qual os cadastros de soluções realizados já reuniam informação suficiente para que fosse implementado um mecanismo de busca na biblioteca. A busca de soluções, então, foi subdividida em uma busca simples (por palavra chave digitada) e uma busca avançada (o usuário procura solução por características específicas). Ao acessar qualquer solução, são apresentadas em tela informações como contexto de uso, categoria em que se enquadra, sua utilização, além de arquivos de mídia explicativos (vídeos, documentos ou imagens) sobre a mesma.

A biblioteca virtual também contou com uma área administrativa, onde são apresentados relatórios de usuários e soluções cadastradas. Nesta área, o administrador pode visualizar informações presentes na biblioteca, publicar notícias e controlar tarefas a serem desenvolvidas no sistema. Ainda, lhe é permitido ver estatísticas acerca dos cadastros realizados, sua quantidade e classificação.

Concluídas todas as páginas que compuseram a biblioteca virtual, e após verificar seu devido funcionamento, deu-se início à construção de um protótipo para o Sistema Operacional Android, planejado com o intuito de oferecer acesso a informações cadastradas no sistema web. Neste aplicativo, o usuário pode acessar seu perfil, visualizar soluções assistivas que cadastrou e realizar busca dentre as soluções existentes na biblioteca, digitando um termo ou palavra chave. A figura 8 exemplifica o funcionamento da versão móvel da biblioteca virtual.

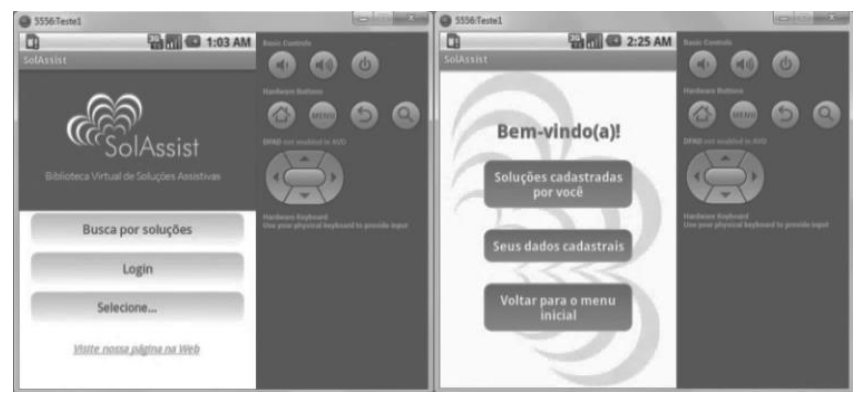

Figura. 8. Tela inicial e perfil do usuário na versão móvel da biblioteca virtual

A biblioteca virtual de soluções assistivas foi implementada de forma responsiva com a utilização do banco de dados MySQL, linguagem de Programação PHP e Javascript. Para o desenvolvimento das interfaces, foi utilizada a linguagem de estruturação HTML5 e folhas de estilo em CSS3. Já o protótipo para Android foi construído no ambiente de desenvolvimento integrado (IDE) Eclipse, com utilização da linguagem de programação Java. A figura 9 demonstra a arquitetura completa posta em prática para a construção e funcionamento da biblioteca virtual de soluções assistivas.

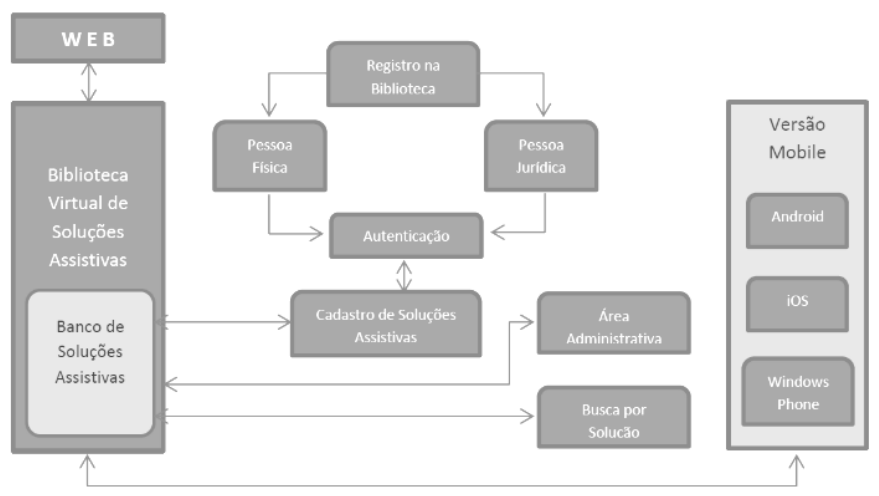

Figura. 9. Arquitetura da biblioteca virtual de soluções assistivas

O projeto de Biblioteca Virtual de Soluções Assistivas, denominado SolAssist, é resultado de atividade de pesquisa em conjunto com a Universidade Federal do Rio Grande do Sul - UFRGS e sua versão atual encontrase disponível na Web através da URL: http://solassist.ufrgs.br/teias/solassistv3/index.php

\section{Resultados Obtidos}

Durante o desenvolvimento do projeto piloto diversos questionários foram enviados com a finalidade de obtenção de dados acerca dos participantes e dos conteúdos apresentados. Dos 34 participantes que iniciaram o 
curso, 10 finalizaram cumprindo a carga horária necessária (no mínimo $75 \%$ de participação) para a obtenção da certificação pela UFRGS como Curso de Extensão.

Um questionário foi disponibilizado antes do início do curso, o que possibilitou a obtenção dos dados a respeito dos referidos participantes relacionados com escolaridade, interesse no curso, local de trabalho, deficiências com as quais atuam, tempo de experiência, área de atuação, classificação do conhecimento em TA. O resultado está apresentado na tabela 02 .

\begin{tabular}{|c|c|c|c|}
\hline \multicolumn{4}{|c|}{$\begin{array}{l}\text { PARTICIPANTES DO MOOC - SOLASSIST } \\
\text { LEARNING - PERFIL }\end{array}$} \\
\hline Idade & Sexo & $\begin{array}{l}\text { Grau de } \\
\text { Instrução }\end{array}$ & \begin{tabular}{|ll} 
Carga & \\
horária & de \\
trabalho &
\end{tabular} \\
\hline $\begin{array}{l}{[21-30]:} \\
06 \% \\
{[31-40]:} \\
45 \% \\
{[41-50]:} \\
35 \% \\
{[51-60]:} \\
14 \%\end{array}$ & $\begin{array}{l}\text { Fem.: } 91 \% \\
\text { Mas.: } 09 \%\end{array}$ & $\begin{array}{l}\text { Curso } \\
\text { técnico 6\% } \\
\text { Graduação } \\
: 39 \% \\
\text { Pós } \\
\text { Graduação } \\
: 55 \%\end{array}$ & \begin{tabular}{|l}
31 a 40 \\
horas: $77 \%$ \\
Mais de 41 \\
horas: $23 \%$
\end{tabular} \\
\hline $\begin{array}{l}\text { Horas diárias } \\
\text { de acesso a } \\
\text { internet }\end{array}$ & $\begin{array}{l}\text { Possui } \\
\text { conhecime } \\
\text { nto em } \\
\text { tecnologias } \\
\text { assistivas }\end{array}$ & $\begin{array}{l}\text { Possui } \\
\text { algum tipo } \\
\text { de } \\
\text { deficiência }\end{array}$ & $\begin{array}{l}\begin{array}{l}\text { Onde } \\
\text { utiliza }\end{array} \\
\text { acesso ê } \\
\text { internet }\end{array}$ \\
\hline $\begin{array}{l}\text { Menos de } 01 \\
\text { hora: } 3 \% \\
\text { Entre } 01 \text { e } \\
03 \text { horas: } \\
52 \% \\
\text { Entre } 04 \text { e } \\
05 \text { anos: } \\
29 \% \\
\begin{array}{lll}\text { Mais } & \text { de } 05 \\
\text { anos: } & 16 \% \\
\end{array}\end{array}$ & $\begin{array}{l}\text { Sim: } 19 \% \\
\text { Não: } 81 \%\end{array}$ & $\begin{array}{l}\text { Sim: } 0 \% \\
\text { Não: } 100 \%\end{array}$ & $\begin{array}{l}\text { Em casa: } \\
30 \% \\
\text { Trabalho: } \\
37 \% \\
\text { Ambos: } \\
29 \%\end{array}$ \\
\hline $\begin{array}{l}\text { Dispositivo } \\
\text { de acesso }\end{array}$ & $\begin{array}{l}\text { Finalidade } \\
\text { de acesso }\end{array}$ & Rede social & que utiliza \\
\hline $\begin{array}{l}\text { Computador } \\
58 \% \\
\text { Notebook: } \\
85 \% \\
\text { Celular: } 58 \% \\
\text { Tablet: } 16 \%\end{array}$ & $\begin{array}{l}\text { Lazer: } \\
26 \% \\
\text { Trabalho: } \\
84 \% \\
\text { Estudo: } \\
81 \%\end{array}$ & $\begin{array}{l}\text { Facebook: } 9 \\
\text { Instagran:13 } \\
\text { Whatsapp: } 1 \\
\text { Twitter:10\% } \\
\text { Linkedin:16 } \\
\text { Outros: } 13 \%\end{array}$ & \\
\hline
\end{tabular}

Tabela 02. Formulário de dados a respeito dos participantes no MOOC Solassist Learning

Ao término do curso foi aplicado outro questionário, que tornou possível analisar a eficácia do curso.
Todos os participantes trabalhavam, o que tornou um pouco difícil a continuação de todo o grupo. Um total de 34 pessoas foram selecionadas para a realização do curso. A seleção priorizou pessoas que atuassem no ensino profissionalizante e com pessoas com deficiência. Algumas destas antes de acessar o ambiente do curso já haviam desistido. Alguns participantes relataram dificuldade em trabalhar com as ferramentas tecnológicas utilizadas bem como o ambiente Wikispaces. Ao acessar o ambiente, antes de estar logado no curso é necessário criar um login e senha e estas instruções estão em inglês. Alguns participantes relataram grandes dificuldades com a língua estrangeira. Um total de 10 participantes finalizaram o curso com a carga horária necessária para certificação.

A respeito do tempo dedicado aos estudos, $89 \%$ dos participantes disseram que conseguiram dedicar tempo suficiente para a realização do curso. As principais dificuldades encontradas estão representadas na figura 10.

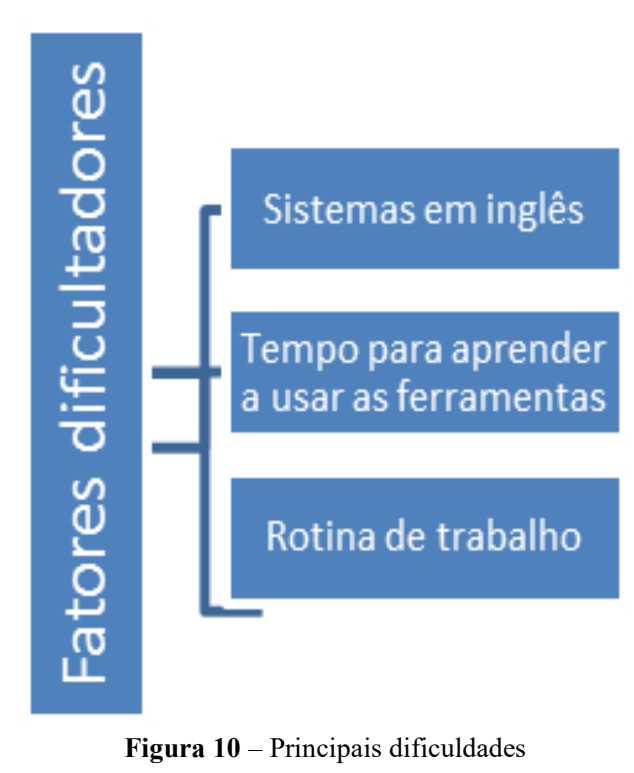

Um total de 33\% dos participantes afirmou que após o curso sugeriu a implantação e utilização de recursos de Tecnologia Assistiva e que realmente puderam ser implantados. Os demais $67 \%$ afirmaram que através do curso puderam perceber onde se aplica os recursos de TA, porém ainda não foram implantados. A figura 11 apresenta os recursos que puderam ser implantados pelos participantes com base nos conhecimentos adquiridos no decorrer do curso. 


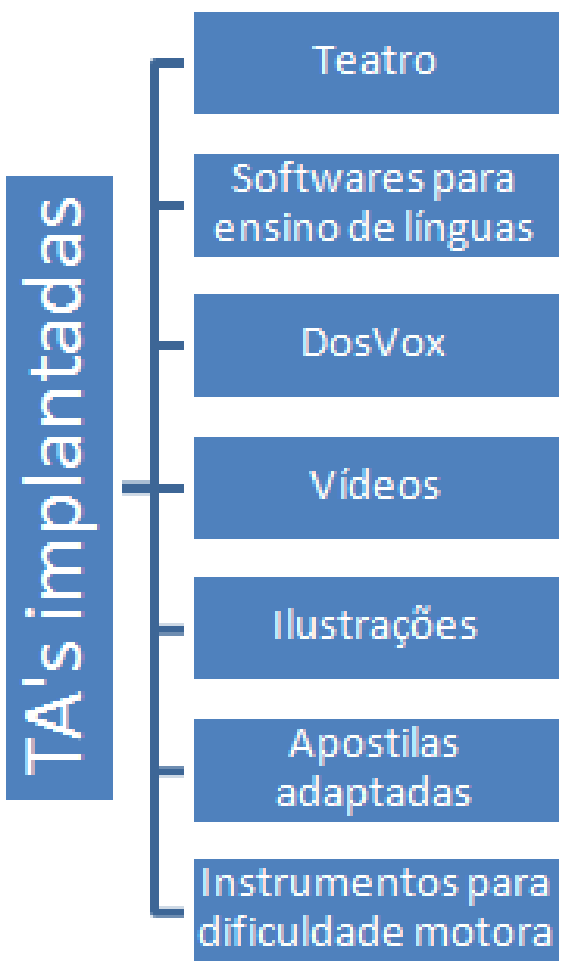

Figura 11 - TAs implantadas

Por meio das respostas aos questionamentos foi possível gerar um panorama geral sobre o público alvo do curso, suas dificuldades e sobre as demandas que foram apresentadas de acordo com as necessidades de adaptações de ambientes de trabalhos e adaptações a serem realizadas.

Os participantes que finalizaram o curso com a carga horária mínima para obtenção do certificado, receberam um certificado expedido pela UFRGS, como forma de curso de extensão.

A realização deste projeto piloto possibilitou a obtenção de um panorama geral. Para que o projeto seja desenvolvido será fundamental a utilização dos dados obtidos até o momento como forma de possibilitar uma formação mais completa aos participantes.

\section{Trabalhos Futuros}

O MOOC Solassist Learning representa um projeto piloto realizado através da plataforma Wikispaces e de recursos educativos disponíveis na Web. A realização deste projeto piloto possibilitou a obtenção de dados que servirão para melhorias do projeto final. Os resultados dos questionários aplicados durante o curso foram analisados e neste momento está sendo desenvolvido um projeto final.
Dentre as principais alterações necessárias para o projeto final é a utilização de conteúdos mais dinâmicos, como vídeos e objetos de aprendizagem e também a utilização de uma plataforma com maior usabilidade e que tenha idioma em português.

\section{Considerações Finais}

Através da notável expansão da Educação a Distância tornam-se necessárias novas metodologias, conceitos e tecnologias que possibilitem o crescimento desta modalidade de estudo, bem como tornem possível o desenvolvimento desta forte demanda.

O MOOC apresentado neste trabalho tem como principal objetivo auxiliar neste desenvolvimento, possibilitando a realização de pesquisas e novos estudos sobre este conceito. A utilização de diferentes plataformas, como o wikispaces, integrado com a biblioteca virtual de Tecnologia Assistiva visa apoiar o desenvolvimento do que conceituamos como MOOC, por utilizar materiais dinâmicos e, mesmo sendo um curso de baixa carga horária, possibilitar links que possam ser explorados mais amplamente de acordo com o interesse de cada estudante.

Ainda, a utilização de uma biblioteca virtual de soluções assistivas, projetada e desenvolvida pelos autores, para servir de suporte prático ao aprendizado, mostrou-se uma ferramenta eficaz e de grande valia, uma vez que possibilita o contato com tecnologias assistivas aplicadas ao dia-a-dia que podem ser vivenciadas em um curso online, como é o caso da integração com o MOOC.

Os resultados apresentados possibilitaram o conhecimento do público cursista, das dificuldades apresentadas no decorrer do curso e das fragilidades do ambiente que podem ser melhoradas para a próxima versão do curso.

Por fim, os resultados apresentados pelos questionários realizados ao término do curso, demonstram que a integração realizada dos diversos materiais, incluindo um ambiente dinâmico e a integração de diferentes plataformas, como a biblioteca virtual (Solassist) possibilitaram uma formação bastante ampla sobre Tecnologia Assistiva aos cursistas.

\section{Referências}

[1] AAATE. Association for the Advancement of Assistive Technology in Europe. 2003. Disponível em: $<\mathrm{http}: / /$ www.atireland.ie/aaate/>. Acesso em: $13 \mathrm{de}$ agosto de 2015.

[2] BERSCH, Rita. (2008) Introdução à Tecnologia Assistiva. CEDI - Centro Especializado em Desenvolvimento Infantil. Porto Alegre - RS. 
[3] EASTIN. (2015) O que é EASTIN? Disponível em: $<$ http://www.eastin.eu/pt-pt/whatIsEastin/index $>$. Acesso em: 16 abr. 2015.

[4] FLICK. Introdução à pesquisa qualitativa. 3 ed. Porto Alegre: Artmed, 2009.

[5] GETITCORP. Disponível em: https://www.getitcorp.tv/. Acesso em março de 2016.

[6] MANZINI, E. J. (2005) Tecnologia assistiva para educação: recursos pedagógicos adaptados. Brasília - DF: Ensaios pedagógicos: construindo escolas inclusivas, p. 82-86.

[7] MCFEDRIES, P. "I'm in the Mood for MOOCs". IEEE SpEctrum. p.30. 2012.

[8] MOTA, INAMORATO(2012) MOOC, uma revolução em curso. Jornal da Ciência. Publicado em 26/11/12

[9] PASSERINO, Liliana Maria ; PEREIRA, A. C. C. Educação, Inclusão e Trabalho: um debate necessário. Educação e Realidade, v. 39, p. 831-846, 2014.

[10] Portal Nacional de Tecnologia Assistiva. (2015) Conheça o Portal. Disponível em: $<h t t p: / / w w w$.assistiva.org.br/conheca-o-portal $>$. Acesso em: 16 abr. 2015.

[11] Portale SIVA. (2015). Quem somos nós. Disponível em: $\quad<$ http://portale.siva.it/it-IT/home/aboutUs>. Acesso em: 16 abr. 2015.

[12] SANTANA, B.; ROSSINI, C.; PRETTO, N. L. (Org.). Recursos Educacionais Abertos: práticas colaborativas e políticas públicas. São Paulo/Salvador: Casa da Cultura Digital/EDUFBA, 2012. p. 17-32. Disponível em: $<$ http://www.artigos.livrorea.net.br/2012/05/educaca o-aberta-configurando-ambientes-praticas-erecursos-educacionais/>. Acesso em: 01 jan. 2014.

[13] SIEMENS, G. MOOCs are really a platform. Elearnspace, July 25, 2012. 